\title{
Ferromagnetic resonance imaging of Co films using magnetic resonance force microscopy
}

\author{
B. J. Suh, P. C. Hammel, ${ }^{\text {a) }}$ and Z. Zhang \\ Condensed Matter and Thermal Physics, Los Alamos National Laboratory, Los Alamos, New Mexico 87545
}

M. M. Midzor and M. L. Roukes

Condensed Matter Physics, California Institute of Technology, Pasadena, California 91125

J. R. Childress

University of Florida, Gainesville, Florida 32611

(Received 21 January 1998; accepted 21 May 1998)

\begin{abstract}
Lateral one-dimensional imaging of cobalt $(\mathrm{Co})$ films by means of microscopic ferromagnetic resonance (FMR) detected using the magnetic resonance force microscope (MRFM) is demonstrated. A novel approach involving scanning a localized magnetic probe is shown to enable FMR imaging in spite of the broad resonance linewidth. We introduce a spatially selective local field by means of a small, magnetically polarized spherical crystallite of yttrium iron garnet (YIG). Using MRFM-detected FMR signals from a sample consisting of two Co films, we can resolve the $\sim 20 \mu \mathrm{m}$ lateral separation between the films. The results can be qualitatively understood by consideration of the calculated spatial profiles of the magnetic field generated by the YIG sphere.

(C) 1998 American Vacuum Society. [S0734-211X(98)08204-3]
\end{abstract}

\section{INTRODUCTION}

Magnetic field sensors comprised of layered magnetic materials are having a significant impact on magnetic recording technologies. The sensitivity of these layered materials to characteristics of the buried interfaces between layers highlights the need for a high resolution, spatial imaging probe of structural and magnetic properties of materials. The magnetic resonance force microscope (MRFM) can potentially fill this need. MRFM detection of both nuclear magnetic resonance $(\mathrm{NMR})^{1}$ and ferromagnetic resonance $(\mathrm{FMR})^{2}$ has been demonstrated. Each of these has advantages for microscopic imaging in magnetic materials. FMR benefits from very high signal sensitivity because it couples to fully polarized electronic moments, and conventional FMR has a demonstrated capability for determining crucial magnetic properties such as magnetic anisotropies of the thin films and the magnetic exchange coupling between nearby ferromagnetic layers. ${ }^{3}$ However, microscopic FMR imaging cannot be performed using conventional techniques because conventional FMR is performed in a uniform magnetic field so there is no means to identify the spatial origin of a particular contribution to the FMR signal.

Magnetic resonance imaging employs a magnetic field gradient to identify the spatial origin of a resonance signal. Through the magnetic resonance condition $\left(\omega_{0}=\gamma H_{0}\right.$ for a noninteracting spin having gyromagnetic ratio $\gamma$, where $H_{0}$ is the applied field) the field gradient allows the spatial origin of the signal to be inferred from the resonance frequency. This assumes that the resonance frequency $\omega_{0}$ is a local function of applied field $H$, that is, $\omega_{0}(\mathbf{r})=f[H(\mathbf{r})]$. Because of strong dipole couplings to neighboring spins in a

a) Author to whom correspondence should be addressed; electronic mail: pch@lanl.gov ferromagnet, the resonance frequency at a particular spatial location is nonlocal, i.e., it is determined by magnetization of neighboring regions in addition to the value of the field applied at that point. Thus, imaging by means of an applied field gradient is not as straightforward as in the case of noninteracting spins, such as occurs in NMR.

Here we address two aspects of this problem. First, we demonstrate an alternative approach to imaging using a spatially localized magnetic field source, and we present a scanning FMR image in a Co film obtained using a small yttrium iron garnet (YIG) grain as the magnetic probe. This approach is similar to that used in magnetic force microscopy (MFM) ${ }^{4}$ where only the spin magnetization in the vicinity of the probe tip contributes to the signal. Spatial resolution in this approach is determined by the extent of the field produced by the magnetic probe, and this approach is not expected to yield resolution superior to that of the MFM. ${ }^{4}$ However, FMR imaging has the advantage that it can provide microscopic determination of quantities not obtainable through MFM measurements such as the interlayer exchange coupling. Second, the field gradient due to the YIG particle is sufficiently large that we are able explore the affect of an applied field gradient on a ferromagnetic film with broad intrinsic linewidth and show that it can cause spatially separate regions of a contiguous film to resonate at distinct frequencies, thus indicating that imaging by means of an applied gradient is possible.

The MRFM mechanically detects the magnetic resonance signal by sensitively detecting the oscillatory response of a micromechanical resonator. ${ }^{1,5-7} \mathrm{~A}$ small permanent magnet is used to produce a spatially inhomogeneous magnetic field which plays two crucial roles. First it establishes the coupling

$$
\mathbf{F}=(\mathbf{m} \cdot \boldsymbol{\nabla}) \mathbf{B}
$$


between the spin magnetization $\mathbf{m}$ and the mechanical element. Second, it enables imaging as discussed earlier. The irradiation frequency $\omega_{\text {rf }}$ defines a surface of constant field called the "sensitive slice" in which the magnetic resonance condition is met, that is, in which $\omega_{0}=\omega_{\mathrm{rf}}$; only those electron spins in this slice will couple to the rf field. Modulating the sample magnetization at the resonance frequency of the mechanical resonator drives it into oscillation; this is accomplished by modulating either the rf field intensity or the applied magnetic field, or both. The resultant time-varying force will be due only to the spins within the sensitive slice. The resonant oscillation of the cantilever is detected by means of an optical fiber interferometer. Images are obtained by scanning the sensitive slice throughout the sample.

In principle, the spatial resolution is given by the sensitive slice width $\Delta z$ which is determined by the intrinsic resonance linewidth $\Delta H_{\mathrm{lw}}$ and the applied field gradient $\partial H_{0} / \partial z$ :

$$
\Delta z \simeq \frac{\Delta H_{1 \mathrm{w}}}{\partial H_{0} / \partial z} .
$$

An additional requirement is that signal detection sensitivity must be sufficient to observe the signal from the resolved volume. We have demonstrated earlier ${ }^{2}$ that the sensitivity of MRFM detected FMR in YIG films is sufficient to enable studies of microscopic volumes. However, the FMR lines remained sharp in spite of the application field gradient sufficiently large that our sensitive slice width [given by Eq. (2)] should have been smaller than the sample size. That is, the gradient should have been sufficient to broaden the line. Clearly then, nonlocal effects due to dipole couplings dominate over the applied field gradient in this case. Similarly, Wago et al. ${ }^{8}$ found that imaging in YIG by standard means involving an applied field gradient was not successful. This contrasts with successful demonstrations of microscopic MRFM imaging by means of both electron spin resonance (ESR) and NMR. ${ }^{9,10}$

We have recently focused our efforts on studies of Co films ${ }^{11}$ whose FMR lines are even broader than YIG, making the task more difficult. Here we explore the requirements for spatial imaging of magnetic properties of Co films using magnetic resonance force microscopy.

\section{EXPERIMENTAL DETAILS}

\section{A. Sample}

A commercially available atomic force microscopy cantilever ${ }^{12}$ serves as the resonant mechanical element used to detect the magnetic resonance signal. Two laterally separated Co films were placed on the cantilever by sputter deposition through a mask consisting of two $70 \pm 5 \mu \mathrm{m}$ wide slits separated by $20 \pm 5 \mu \mathrm{m}$. Because one of the slits only partially overlapped the end of the cantilever the resulting sample geometry, starting at the free end of the cantilever, is approximately [20 $\mu \mathrm{m}$ (Co) $\mid 20 \mu \mathrm{m}$ (separation) $\mid 70 \mu \mathrm{m}$ (Co)]. Because the mask was not in direct contact with the cantilever surface, the film edges were not vertical. Each

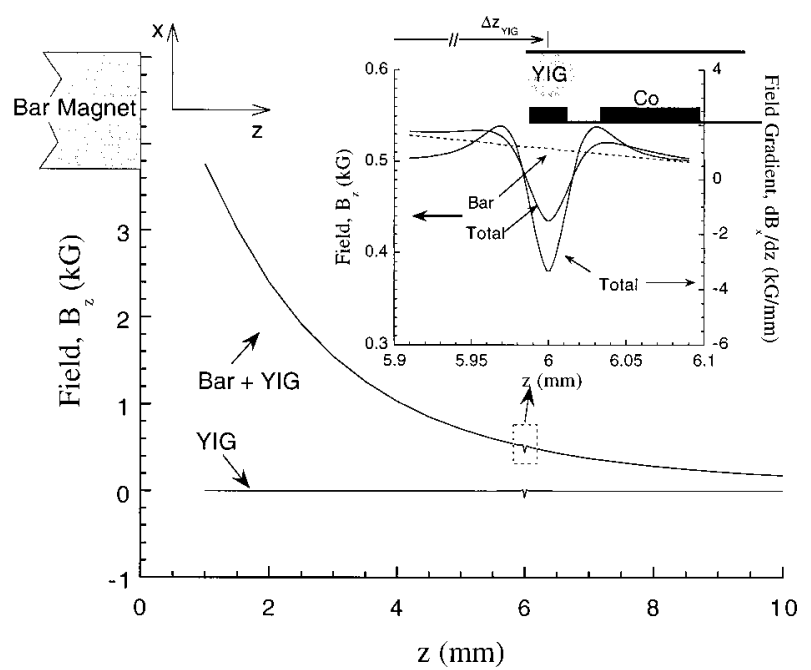

FIG. 1. Calculated field and field gradient profiles for the YIG sphere. A schematic diagram of the relative positions of the YIG sphere and the Co films is shown.

section of Co film is $\approx 600 \AA$ thick and is protected by $\mathrm{Ag}$ layers above and below; the vertical profile is then [ $\mathrm{Si}$ (cantilever) | Ag (35 ̊) | Co $(600 \AA) \mid \operatorname{Ag}(70 \AA)]$.

\section{B. Measurements}

The experiments were performed with the field applied in the Co film plane which allows small saturation and therefore, resonance fields, of order hundreds of Gauss. ${ }^{11}$ A schematic illustration of the arrangement is shown in Fig. 1. The rf irradiation at a frequency, $\omega_{\mathrm{rf}} / 2 \pi \simeq 7.9 \mathrm{GHz}$, was generated by microstrip resonator. ${ }^{11,13,14}$ The cantilever resonance frequency was $f_{c} \simeq 12 \mathrm{kHz}$, and its $Q$ value was $\sim 10^{4}$ at 70 mTorr and room temperature.

The sample is positioned slightly off the axis of the bar magnet at $(x, z) \simeq(1$ and $6 \mathrm{~mm})$ with respect to a point at the center of the near face of the bar magnet (see Fig. 1). The bar magnet is $6.35 \mathrm{~mm}\left(\frac{1}{4} \mathrm{in}\right.$.) long and $6.35 \mathrm{~mm}\left(\frac{1}{4} \mathrm{in}\right.$.) in diameter. The field from an electromagnetic solenoid is scanned from -300 to $300 \mathrm{G}$.

An approximately spherical YIG grain $\approx 30 \mu \mathrm{m}$ in diameter is mounted on a second cantilever and then scanned above the sample with a fixed vertical separation $\Delta x$ $\sim 30 \mu \mathrm{m}$ as indicated in Fig. 1. The horizontal position (i.e., the $z$ axis position) of the YIG grain with respect to the Co films is denoted by $\Delta z_{\mathrm{YIG}}$ with respect to an arbitrary reference as illustrated in Fig. 1.

Measurements were performed both by selecting a position for the YIG sphere $\left(\Delta z_{\mathrm{YIG}}\right)$, then sweeping the solenoid field, and by scanning the YIG sphere horizontally across the two films (i.e., varying $\Delta z_{\mathrm{YIG}}$ ) at a fixed value of the solenoid field. 


\section{RESULTS AND DISCUSSION}

\section{A. YIG sphere magnetic field profile}

Figure 1 shows the calculated spatial variation of the magnetic field and the field gradient of the YIG sphere. From Eq. (1) the appropriate expression for our geometry is

$$
F_{x}=m_{x} \frac{\partial B_{x}}{\partial x}+m_{z} \frac{\partial B_{x}}{\partial z}
$$

where $m_{\alpha}$ refers to the magnetization of the Co film, and $B_{\alpha}$ to the various components of the applied field. Since the field due to the solenoid and bar magnet is nearly parallel to $\hat{z}$, that is, in the plane of the Co film, $m_{z} \gg m_{x}$, the first term in Eq. (3) can be neglected. The calculated results for $B_{z}$ and $\partial B_{x} / \partial z$ are shown in Fig. 1 .

YIG was chosen as a probe magnet because it has a small saturation magnetization value, $4 \pi M_{s}=1.6 \mathrm{kG}$ which allows it to be easily saturated in small applied fields. Therefore its magnetization does not change in response to variation of the external field due to field sweeping or displacement of the YIG grain with respect to the bar magnet.

We can expect that FMR signals arising from sample regions affected by the field of the YIG sphere will have three characteristics:

(1) As shown in the inset of Fig. 1, the YIG sphere locally reduces the field by $\sim 100 \mathrm{G}$. Thus, a correspondingly higher applied field will be required to meet the resonance condition, so the resonance spectrum from the region affected by the local field generated by the YIG sphere will appear at higher solenoid field.

(2) The signal strength in a MRFM is enhanced by a larger field gradient. ${ }^{15,16}$ The larger magnitude of the field gradient of the YIG sphere will then enhance the size of the signals originating from Co experiencing the field of the sphere.

(3) The sign of the gradient from the YIG sphere is reversed from that of the bar magnet; this will shift the phase of the signals originating from Co near the sphere by $\pi$ relative to signals from other regions.

\section{B. FMR spectra and scanning image}

Figure 2 shows a series of in-phase FMR/MRFM spectra obtained by scanning the solenoid field at several values of $\Delta z_{\mathrm{YIG}}$. A single FMR signal with a resonance linewidth $\approx 60 \mathrm{G}$ is observed when the YIG sphere is located far from the sample region. The magnetic field gradient $\partial B_{x} / \partial z$ due to the bar magnet at the sample is $\sim 0.2 \mathrm{G} / \mu \mathrm{m}$; this corresponds to a field difference of less than $20 \mathrm{G}$ across the sample. This is smaller than the observed resonance linewidth $(\approx 60 \mathrm{G})$ so the field gradient is too small to resolve the two films laterally separated by $\sim 20 \mu \mathrm{m}$.

When the YIG sphere approaches the sample region, an additional signal at higher field begins to appear. The maximum shift of the additional signal with respect to the original one is $\approx 170 \mathrm{G}$. This is larger than the calculated value $(\sim 100 \mathrm{G}$; see the inset of Fig. 1) of the additional field

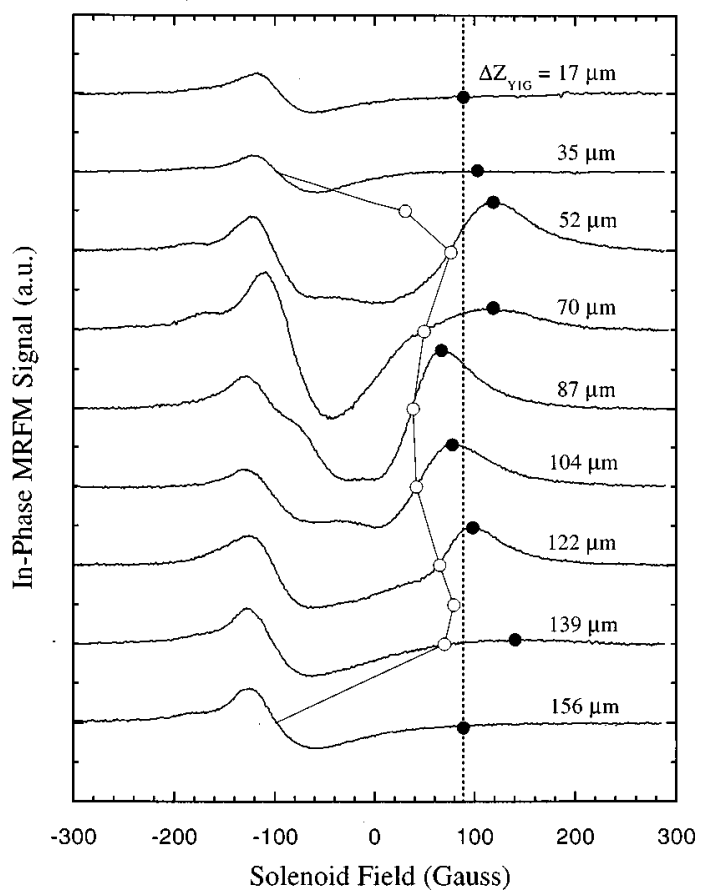

FIG. 2. Series of FMR spectra as a function of solenoid field are shown for several values of $\Delta z_{\mathrm{YIG}}$ : the positions of the maximum deviation (O) and the center of the additional signal $(O)$ are shown. The dotted line indicates the value of the solenoid field at which the signal intensity as a function of $\Delta z_{\text {YIG }}$ (shown as a dotted line in Fig. 3) is determined.

contributed by the YIG sphere. The error in the calculation is ascribed to uncertainties in the size and shape of the YIG grain and the vertical distance of the YIG from the sample $(\Delta x)$. For $\Delta z_{\text {YIG }}$ near $70 \pm 10 \mu \mathrm{m}$, the additional signal is suppressed indicating the YIG sphere is between the two films. The intensity of the additional signal recovers when the YIG sphere is moved over the second Co film.

The additional signals generated by the YIG sphere shown in Fig. 2 have the expected features discussed in Sec. III A above, including the characteristic $\pi$ phase shift. A more detailed analysis of the dependence of the signal intensity and of the spectral shift of the additional signal on $\Delta z_{\mathrm{YIG}}$ provides the basis for this approach to imaging the Co films.

The dependence of the MRFM signal strength $S\left(\Delta z_{\mathrm{YIG}}\right)$ on the position $\Delta z_{\text {YIG }}$ of the YIG sphere was extracted in two ways. First, $S\left(\Delta z_{\mathrm{YIG}}\right)$ was determined at a fixed value of the solenoid field, $B=90 \mathrm{G}$ (the average value of the peak position of the additional signals) shown as a dotted line in Fig. 2. $S\left(\Delta z_{\mathrm{YIG}}\right)$ obtained in this way is shown as a dotted curve in Fig. 3. The amplitude of the additional signal is approximately determined by the area of the Co film affected by the selective local field generated by the YIG sphere. Therefore, $S\left(\Delta z_{\mathrm{YIG}}\right)$ gives the lateral spatial profile of the Co sample. Two regions are clearly distinguished from the dotted curve in Fig. 3: one is $\sim 20 \mu \mathrm{m}$ wide and the other is $\sim 60 \mu \mathrm{m}$ wide separated by $\sim 15 \mu \mathrm{m}$. This is in reasonable agreement with the actual sample profile, [20 $\mu \mathrm{m}|20 \mu \mathrm{m}| 70 \mu \mathrm{m}]$. The amplitude of the first region is observed to be small compared to the second one. We attribute this to a misreading of the sig- 


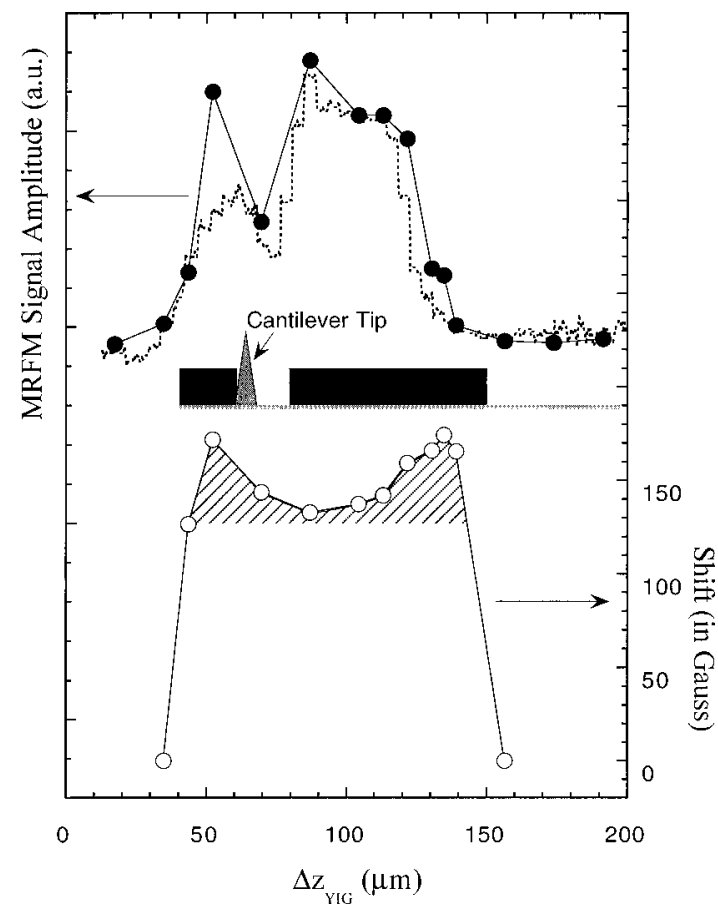

FIG. 3. (Upper) Dotted curve shows the variation of the signal amplitude as a function of the position of the YIG sphere, $\Delta z_{\mathrm{YIG}}$, at a fixed value of solenoid field $B=90 \mathrm{G}$. The curve with solid circles shows the variation of maximum deviation of the additional signal. (Lower) Curve with open circles indicates the magnetic field shift of the additional signal with respect to the position of the original signal. The estimated sample profile obtained from knowledge of the shadow mask dimensions and optical microscopy on the Co sample is illustrated in the middle of the figure for comparison.

nal amplitude resulting from shifts in the field at which the peak occurs. In fact, an alternative approach to determining $S\left(\Delta z_{\mathrm{YIG}}\right)$ in which it is extracted from the peak amplitude of the additional signal (marked as solid circles in Fig. 2) rather than at a constant value of applied field, gives the results shown by solid circles in Fig. 3. Using this approach, the amplitudes of the signals from the two films are more similar.

These shifts in peak position of the additional signal (open circles in Fig. 2) are expected as discussed in Sec. III A. The signal shape ${ }^{16}$ from a sample whose dimensions are smaller than the sensitive slice width is a derivative of the magnetic resonance response. Therefore the center resonance spectrum will correspond to the point where this derivative vanishes. ${ }^{16}$ Although the gradient is sufficiently large that this is no longer strictly appropriate, we determine the resonance field from the zero crossing of the signal. These results are shown as open circles in Figs. 2 and 3. We expect that any region directly adjacent to the YIG sphere will experience the maximum negative local field and therefore the maximum shift. As the sphere is laterally displaced away from the Co film, the Co sample will experience first the shoulder of the selective local field (see the inset of Fig. 1), giving rise to the smaller shift of the additional signal, then, when no additional field is experienced the shift will vanish. Therefore, the value of the shift also represents the sample profile. However, since the magnitude of the local field is sensitive to the vertical distance of the YIG sphere from the sample due to its small size, the uncertainty in the separation $\Delta x$ of the YIG sphere from the sample as the sphere is scanned along the sample cantilever can introduce error. The sample profile deduced from the value of the shift as a function of $\Delta z_{\mathrm{YIG}}$ is shown by open circles in Fig. 3. The poorer agreement with the known sample profile is attributed to uncertainty in $\Delta x$.

A final point is that the additional signals associated with the YIG sphere in several of the spectra in Fig. 2 are clearly broadened by the application of the large applied field gradient of the YIG sphere. This demonstrates that imaging by means used in conventional magnetic resonance imaging (MRI) is possible in FMR. These results also provide an indication that, even with the modest gradients $(<10 \mathrm{G} / \mu \mathrm{m})$ used here, resolution on the scale of tens of microns can be achieved in FMR imaging with conventional MRI techniques.

\section{SUMMARY}

We have presented FMR spectra which demonstrate scanning FMR imaging using the MRFM for the first time. By scanning a selective local field generated by a small YIG sphere, we are able to reconstruct the lateral sample profile along the $\hat{z}$ axis $(20 \mu \mathrm{m}|15 \mu \mathrm{m}| 60 \mu \mathrm{m})$, which should be compared with the profile expected on the basis of the mask used for depositing the sample: $(20 \mu \mathrm{m}|20 \mu \mathrm{m}| 70 \mu \mathrm{m})$.

Unlike conventional MRI where the resolution is determined by the strength of the applied field gradient [Eq. (2)], this approach has the disadvantage that the resolution is determined by the dimensions of the probe magnet. Although resolution superior to that of MFM is not expected, FMR imaging offers distinct advantages in that magnetic properties such as the interlayer exchange coupling can be microscopically mapped. The resolution using the present approach can be improved significantly by using a smaller magnetic probe and by improved control over the distance separating the probe and sample as the probe is scanned. Finally, we have for the first time, observed a broadening of the FMR linewidth arising from the field gradient of the small magnetic particle. This indicates that conventional magnetic resonance imaging techniques can be applied in FMR imaging. Studies to determine the limits of resolution using conventional MRI techniques for FMR imaging are underway.

\section{ACKNOWLEDGMENTS}

Work at Los Alamos National Laboratory was performed under the auspices of the U.S. Department of Energy. One of us (Z.Z.) acknowledges the support of the Center for Nonlinear Studies at Los Alamos National Laboratory.

${ }^{1}$ D. Rugar, O. Züger, S. T. Hoen, C. S. Yannoni, H.-M.Vieth, and R. D. Kendrick, Science 264, 1560 (1994).

${ }^{2}$ Z. Zhang, P. C. Hammel, and P. E. Wigen, Appl. Phys. Lett. 68, 2005 (1996).

${ }^{3}$ P. E. Wigen, in Magnetic Multilayers, edited by L. H. Bennett and R. E. Watson (World Scientific, Singapore, 1994), pp. 183-226.

${ }^{4}$ D. Rugar, H. J. Mamin, P. Guethner, S. E. Lambert, J. E. Stern, I. McFadyen, and T. Yogi, J. Appl. Phys. 68, 1169 (1990). 
${ }^{5}$ J. A. Sidles, Appl. Phys. Lett. 58, 2854 (1991).

${ }^{6}$ J. A. Sidles, Phys. Rev. Lett. 68, 1124 (1992).

${ }^{7}$ P. C. Hammel, Z. Zhang, G. J. Moore, and M. L. Roukes, J. Low Temp. Phys. 101, 59 (1995).

${ }^{8}$ K. Wago, D. Botkin, C. S. Yannoni, and D. Rugar (preprint, 1998).

${ }^{9}$ O. Züger and D. Rugar, J. Appl. Phys. 75, 6211 (1994).

${ }^{10}$ O. Züger, S. T. Hoen, C. S. Yannoni, and D. Rugar, J. Appl. Phys. 79, 1881 (1996)

${ }^{11}$ Z. Zhang et al. (preprint, 1998).
${ }^{12}$ Digital Instruments, 520 E. Montecito St, Santa Barbara, CA. The experiments reported here use type ESP cantilevers which are $450 \mu \mathrm{m}$ long, $30-40 \mu \mathrm{m}$ wide and $1-3 \mu \mathrm{m}$ thick.

${ }^{13}$ W. J. Wallace and R. H. Silsbee, Rev. Sci. Instrum. 62, 1754 (1991).

${ }^{14} \mathrm{~K}$. Wago, O. Züger, J. Wegener, R. Kendrick, C. S. Yannoni, and D. Rugar, Rev. Sci. Instrum. 68, 1823 (1997).

${ }^{15}$ J. A. Sidles and D. Rugar, Phys. Rev. Lett. 70, 3506 (1993).

${ }^{16}$ Z. Zhang, M. L. Roukes, and P. C. Hammel, J. Appl. Phys. 80, 6931 (1996). 